\title{
GENOTIPIFICACIÓN DE CEPAS DE Listeria monocytogenes AISLADAS DE LECHE CRUDA DE DIFERENTES GANADERÍAS DE LIMA MEDIANTE EL ADN POLIMÓRFICO AMPLIFICADO AL AZAR
}

\author{
ODALIS ESTRADA-RAMOS',2 Y AMPARO I. ZAVALETA
}

'CLEIBA Y 'LAB. BIOLOGIA MOLECULAR. FACULTAD DE FARMACIA Y BIOQUIMICA DE LA UNIVERSIDAD NACIONAL. MAYOR DE SAN MARCOS

\begin{abstract}
RESUMEN
En el presente estudio se determinó la variabilidad genética de cepas de Listeria monocytogenes aisladas de leche cruda procedentes de 11 ganaderías lecheras que expenden este producto alimenticio en Lima. Se tomaron 250 unidades de muestra equivalentes a 50 muestras de leche cruda según las indicaciones de la FDA y se sembraron en los medios especificos Oxford y Palcam. L. monocytogenes fue aislada en el $8 \%$ (4/50) de las muestras analizadas procedentes dos de Puente Piedra y dos de Lurín. En el $4 \%(2 / 50)$ de las muestras se encontró Listeria spp. Se aislaron 4 colonias por muestra positiva obteniendo un total de 16 aislados de L. monocytogenes, éstos fueron identificados por pruebas bioquímicas y la reacción en cadena de la polimerasa. Los aislados de L. monocyiogenes fueron genotipificados por la técnica del ADN polimórfico Amplificado al Azar (RAPD) utilizando 10 cebadores de secuencia arbitraria. Los perfiles de RAPD fueron muy homogéneos en las 16 cepas, generando tan sólo tres genotipos denominados I, II y III a los cuales pertenecen 4,10 y 6 cepas respectivamente, similares genotipos se obtuvieron en cepas de L. monocytogenes procedentes de muestras clínicas de humanos. El $62.5 \%$ (10/16) de cepas de L. monocytogenes aisladas de leche cruda de las ganaderias de Lima se agrupan mediante la técnica del RAPD en el genotipo II.
\end{abstract}

Palabras clave: Listeria mcnocytogenes, leche cruda, PCR, RAPD, ganaderias lecheras, Lima.

\begin{abstract}
SUMMARY
In the present study it was determined the genetic variabilify of Listeria monocylogenes strains isolated of raw milk from 11 dairy farms that sell this nutritional product in Lima. 250 units of sample equivalent to 50 raw milk samples were taken according to FDA rules and they were streaked on the specific Oxford and Palcam media. L. monocytogenes was isolated in $8 \%(4 / 50)$ samples, 2 of Puente Piedra and 2 of Lurin. In $4 \%(2 / 50)$ of the samples was got Listeria spp. By positive sample 4 colonies were taken obtaining a total of $16 \mathrm{~L}$. monocytogenes isolates, these were identified by biochemical tests and the polymerase chain reaction. L. monocytogenes strains were genotyping by the technique of the Random Amplified Polimorphic DNA (RAPD) using 10 primers of arbitrary sequence. The RAPD profiles were very homogenous in the 16 isolates, generating only three genotypes named I, II and III to which belong 4,10 and 6 isolates respectively, similar genotypes were obtained in $L$. monocytogenes strains coming from clinical samples of humans. The $62,5 \%(10 /$ 16) of $L$. monocytogenes strains isolated of raw milk of dairy farms from Lima are grouped by RAPD technique in genotype II.
\end{abstract}

Key words: Listeria monocytogenes, raw milk, PCR, RAPD, dairy farms, Lima

\section{INTRODUCCIÓN}

Listeria monocytogenes es un bacilo Gram positivo, adquirido por medio de los alimentos y causante de meningitis, septicemia, encefalitis, abortos, partos prematuros que el $30 \%$ de casos son mortales, afecta preferentemente a gestantes, recién nacidos, ancianos y a individuos con el sistema inmune debilitado o con tratamientos prolongados (1).

L. monocytogenes es un microorganismo muy peculiar ya que resiste a condiciones ambientales extremas de temperatura, concentración de $\mathrm{O}_{2} \mathrm{y}$ $\mathrm{pH}$. La capacidad de la bacteria para sobrevivir en el interior o en la superficie del alimento en condiciones adversas por tiempos prolongados y crecer a temperaturas bajas de $24^{\circ} \mathrm{C}$ han hecho de L. monocytogenes una preocupación principal para la Salud Pública e Industria agroalimentaria en la última década $(2,3,4)$.

La leche y quesos artesanales constituyen excelentes medios de cultivo y protección para determinados microorganismos sobre todo para las bacterias patógenas cuya multiplicación depende principalmente de la temperatura y de la pre-

* jr. Puno 1002 Lima 1. PERU 
sencia de otros microorganismos competitivos 0 de sus metabolitos. En este tipo de productos se han descrito el mayor número de brotes de listeriosis por lo que son considerados alimentos de alto riesgo y de análisis continuo $(5,6,7,8,9$, $10)$.

El notable desarrollo de técnicas alternativas a las tradicionales, como los métodos de biología molecular, han permitido la identificación y tipificación de $L$. monocytogenes utilizando procedimientos rápidos; sin embargo, algunas de estas têcnicas no son convenientes para análisis rutinario, debido a que requieren equipos sofisticados y personal altamente capacitado. La técnica del ADN polimórfico amplificado al azar (RAPD) presenta un alto poder discriminatorio y un menor grado de dificultad por lo que se ha usado con éxito en la caracterización de $L$. monocytogenes por numerosos investigadores (11, $12,13,14,15,16,17)$.

Con el objetivo de determinar la variabilidad genética en cepas de L. monocytogenes aisladas de leche cruda procedentes de los principales establos de Lima, se aisló esta bacteria en los medios selectivos Oxford y Palcam, se identificó mediante las pruebas bacteriológicas convencionales y la Reacción en Cadena de la Polimerasa (PCR); además se caracterizaron por el RAPD utilizando 10 cebadores de secuencia arbitraria con la finalidad de identificar los genotipos circulantes para una mejor vigilancia y control epidemiológico.

\section{MATERIAL Y MÉTODOS}

Cepas. Listeria monocytogenes ATCC 19118, L, monocytogenes ATCC 15313, Staphylococcus aureus ATCC 25923, Rhodococcus equi ATCC 6939, Escherichia coli ATCC 43887 y 5 cepas de $L$. monocytogenes aisladas de muestras clínicas de humanos denominadas: $1 \mathrm{mc} 1,1 \mathrm{mc} 2, \operatorname{lmc} 3,1 \mathrm{mc} 4$, lmc5.

Toma de Muestras. Se tomaron 50 muestras de leche cruda en diferentes establos del departamento de Lima (Tabla 2). Una muestra estuvo constituida por 5 unidades de 25 mililitros cada una. Estas 250 unidades de muestra se llevaron al laboratorio en recipientes estériles, refrigerados y se procesaron dentro de las 24 horas de colectadas.

Aislamiento de L. monocytogenes. Se utilizó la metodología señalada en el Manual de Bacteriología Analítica de la FDA (Food and Drug Administration) para el aislamiento e identificación de Listeria monocytogenes (18), que consistió básicamente en añadir $25 \mathrm{ml}$ de una mezcla de 5 unidades de leche cruda a $225 \mathrm{ml}$ del caldo de enriquecimiento para Listeria, se homogenizó en un equipo Stomacher durante 30 segundos y se llevó a incubación a $30^{\circ} \mathrm{C}$ por $48 \mathrm{~h}$. Después de este tiempo de incubación se sembró por estría en los agares Oxford y Palcam y se incubó a $35^{\circ} \mathrm{C}$ de $24 \mathrm{~h}$ a $48 \mathrm{~h}$. Se seleccionaron 4 colonias sospechosas por muestra y 2 por medio selectivo; y se sembraron en agar tripticasa soya conteniendo extrac-

Tabla. 1. Caracteristicas de los cebadores utilizados en el RAPD

\begin{tabular}{|l|l|c|c|}
\hline Nombre & \multicolumn{1}{|c|}{ Secuencia } & Tamaño(bases) & $\left.\mathrm{T}_{\mathrm{h}}{ }^{\circ} \mathrm{C}\right)^{*}$ \\
\hline OPA-03 & AGTCAGCCA & 10 & 36 \\
OPA-06 & GGTCCCTGAC & 10 & 36 \\
OPA-17 & GACCGCTTGT & 10 & 36 \\
OMP-01" $^{\mathrm{n}}$ & GTTGGTGGCT & 10 & 36 \\
Daf4 $^{12}$ & CGGCAGCGCC & 10 & 36 \\
P1254 $^{13}$ & CCGCAGCCAA & 10 & 36 \\
M132 $^{17}$ & GAGGGTGGCGGTTCT & 15 & 50 \\
PJ118 $^{14}$ & TGTTCGTGCTGTTTCTG & 17 & 50 \\
PJ108 $^{14}$ & GCTTATTCTTGACATCCA & 18 & 50 \\
M13 $^{16}$ & GTTGTAAAACGACGGCCAGT & 20 & 50 \\
\hline
\end{tabular}

* $T_{\mathrm{h}}=$ Temperatura de hibridación 
Tabla 2. Aislamiento de Listeria monocytogenes de leche cruda según lugar de procedencia y medio selectivo.

\begin{tabular}{|l|c|c|c|c|c|}
\hline Lugar de procedencia & $N^{2}$ de muestras & Agar & Oxford & Agar & Palcam \\
\hline Cañete & 6 & $1 / 6$ & $\left(\mathrm{a}^{*}, \mathrm{~b}^{*}\right)$ & $0 / 6$ & \\
Chancay & 3 & $0 / 3$ & & $0 / 3$ & \\
Chorrillos & 1 & $0 / 1$ & & $0 / 1$ & \\
Huachipa & 6 & $0 / 6$ & & $0 / 6$ & \\
Huacho & 7 & $0 / 7$ & & $0 / 7$ & \\
Huaral & 5 & $0 / 5$ & & $0 / 5$ & \\
Huaycan & 1 & $0 / 1$ & & $0 / 1$ & \\
Lurín & 8 & $2 / 8$ & $(\mathrm{c}, \mathrm{d}, \mathrm{g}, \mathrm{h})$ & $2 / 8$ & $(\mathrm{e}, \mathrm{f}, \mathrm{i}, \mathrm{j})$ \\
Pachacamac & 1 & $0 / 1$ & & $0 / 1$ & \\
Puente Piedra & 6 & $3 / 6$ & $\left(\mathrm{k}, \mathrm{l}, \mathrm{o}, \mathrm{p}, \mathrm{s}^{*}, \mathrm{t}^{*}\right)$ & $2 / 6$ & $(\mathrm{~m}, \mathrm{n}, \mathrm{q}, \mathrm{r})$ \\
Trapiche & 2 & $0 / 2$ & & $0 / 2$ & \\
\hline
\end{tabular}

Las letras minúsculas entre paréntesis indican los nombres de los aislados de Listeria monocytogenes $y\left({ }^{*}\right) L$ inocua por pruebas bacteriológicas

to de levadura al $0.6 \%$ a $30^{\circ} \mathrm{C}$ de 24 a $48 \mathrm{~h}$. se hicieron 3 cultivos sucesivos a cada aislado y se conservaron con glicerol al $20 \%$ y en congelación.

Pruebas bacteriológicas. De acuerdo a las recomendaciones del Manual de Bacteriología Analítica (Bacteriological Analytical Manual: BAM) (18) se hicieron las siguientes pruebas: Tinción Gram, movilidad, catalasa, nitratos, hemólisis, fermentación de carbohidratos (glucosa, esculina, maltosa, ramnosa, manitol y xilosa) y la prueba de Christie, Atkins Munch-Peterson (CAMP).

PCR. Los aislados identificados como $L$. monocytogenes por pruebas bacteriológicas se cultivaron en caldo tripticasa soya a $35^{\circ} \mathrm{C}$ durante $18 \mathrm{~h}$, se cosecharon por centrifugación y se extrajo y purifico el ADN genómico según el método descrito por Zavaleta y col.(19) La técnica de la reacción en cadena de la polimerasa (PCR) se realizó usando los siguientes iniciadores descritos: $5^{\prime}$-TTATACGCGACCGAAGCCAAC-3' y 5'CAAACTGCTAACACAGCTACT-3' diseñados a partir del gen iap (20).

El volumen final de reacción fue de $50 \mu$ conteniendo buffer PCR $1 \mathrm{X}, \mathrm{MgCl}_{2} 1,5 \mathrm{mM}$, deoxinucleótido trifosfato $200 \mu \mathrm{M}, \mathrm{Taq}$ ADN polimerasa $1 \mathrm{U}$, iniciadores $20 \mathrm{pmol}$ de cada uno y ADN genómico $50 \mathrm{ng}$. La amplificación del ADN se hizo en el termociclador Perkin Elmer 2400 y las condiciones de reacción fueron las siguientes: Desnaturalización inicial de $95^{\circ} \mathrm{C}$ por $3 \mathrm{~min}$., seguido de 35 ciclos (desnaturalización $94^{\circ} \mathrm{C}$ por
45 seg., hibridación $55^{\circ} \mathrm{C}$ por 45 seg. y polimerización a $72^{\circ} \mathrm{C}$ por $45 \mathrm{seg}$.) y 1 ciclo final de polimerización de $72^{\circ} \mathrm{C}$ por 7 minutos.

Se utilizaron como controles negativos ADN de Escherichia coli ATCC 43887, y como control positivo ADN de Listeria monocytogenes ATCC 19118., L. monocytogenes ATCC 15313 y de cinco cepas de L. monocytogenes procedentes de muestras clínicas de humanos. Los productos de PCR se sometieron a electroforesis en gel de agarosa al 1.5\% utilizando buffer tris- borato-EDTA.

RAPD. Se utilizaron 10 cebadores de secuencia arbitraria descritos previamente cuyas características se presentan en la tabla 1 . Las condiciones de reacción fueron similares a las de la PCR antes descrita, a excepción de la temperatura de hibridación que varió según el tamaño del cebador y la concentración de $\mathrm{MgCl}_{2}$ que cambio de $1.5 \mathrm{mM}$ a $2.5 \mathrm{mM}$.

Los productos de PCR se separaron por electroforesis en geles de agarosa al $1.5 \%$ en buffer TBE 1X usando como marcadores de peso molecular Hyperladder I y II (Bioline Inc.).

Análisis de datos. Los perfiles de ADN fueron registrados en una cámara fotográfica polaroid y analizados visualmente. Los perfiles de RAPD se consideraron idénticos o diferentes en base al'número y al tamaño de los fragmentos de ADN observados en las fotografías obtenidas de los geles para cada cebador. 


\section{RESULTADOS}

\section{Aislamiento e identificación de $L$. monocytogenes}

En los medios selectivos Palcam y Oxford se aisló L. monocytogenes en $4 / 50$ muestras de leche cruda analizadas (Tabla 1). El medio Oxford también permitió aislar L. innocua (según las pruebas bacteriológicas) en 2 muestras de leche adicionales a las anteriores.

L. monocytogenes, bacteria emergente fue aislada en el $8 \%$ de muestras de leche cruda procedentes de diferentes establos productores de Lima (Tabla 2). De las 4 muestras positivas para $L$. monocytogenes se obtuvieron 16 aislados que presentaron características morfológicas y culturales similares a L. monocytogenes de colección y a las de origen clínico. Todos los aislados presen- taron reacción positiva para Gram, catalasa, CAMP, movilidad, $\beta$ hemólisis e hidrólisis de ramnosa, maltosa, esculina, glucosa pero no hidrolizaron manitol ni xilosa.

Las cepas de L. monocytogenes fueron confirmadas por PCR utilizando cebadores específicos diseñados a partir del gen iap descritos por Bubert y col. 1999. El producto amplificado midió 660 pb para todas las cepas de L. monocytogenes aisladas y de colección.

\section{Genotipificación de L. monocytogenes}

Se utilizo el RAPD con 10 cebadores de secuencia arbitraria previamente descritos (tabla 1), cada cebador produjo perfiles de bandas diferen-

Tabla 3. Pruebas bioquímicas y moleculares utilizadas en la identificación y caracterización de Listeria monocytogenes

\begin{tabular}{|cccccccccc|}
\hline Aislado & Xil & Man & Ram & Mal & Esc & Gluc & B-hemo PCR & RAPD \\
\hline a & - & - & - & + & + & + & - & - & nd \\
b & - & - & - & + & + & + & - & - & nd \\
c & - & - & + & + & + & + & + & + & I \\
d & - & - & + & + & + & + & + & + & I \\
e & - & - & + & + & + & + & + & + & I \\
f & - & - & + & + & + & + & + & + & I \\
g & - & - & + & + & + & + & + & + & II \\
h & - & - & + & + & + & + & + & + & II \\
i & - & - & + & + & + & + & + & + & II \\
$\mathrm{j}$ & - & - & + & + & + & + & + & + & II \\
$\mathrm{k}$ & - & - & + & + & + & + & + & + & III \\
l & - & - & + & + & + & + & + & + & III \\
$\mathrm{m}$ & - & - & + & + & + & + & + & + & II \\
$\mathrm{n}$ & - & - & + & + & + & + & + & + & II \\
$\mathrm{o}$ & - & - & + & + & + & + & + & + & II \\
$\mathrm{p}$ & - & - & + & + & + & + & + & + & II \\
$\mathrm{g}$ & - & - & + & + & + & + & + & + & II \\
$\mathrm{r}$ & - & - & + & + & + & + & + & + & II \\
$\mathrm{s}$ & - & - & - & + & + & + & - & - & nd \\
$\mathrm{t}$ & - & - & - & + & + & + & - & - & nd \\
\hline
\end{tabular}

Xil, Xilosa; Man, manosa; Ram, ramnosa; Mal, maltosa; Esc, esculina; Glu, glucosa; B-hemo, beta-hemólisis; PCR, cebadores específicos para Lmonocytogenes, nd, no determinado; +, reacción positiva; -, reacción negativa 


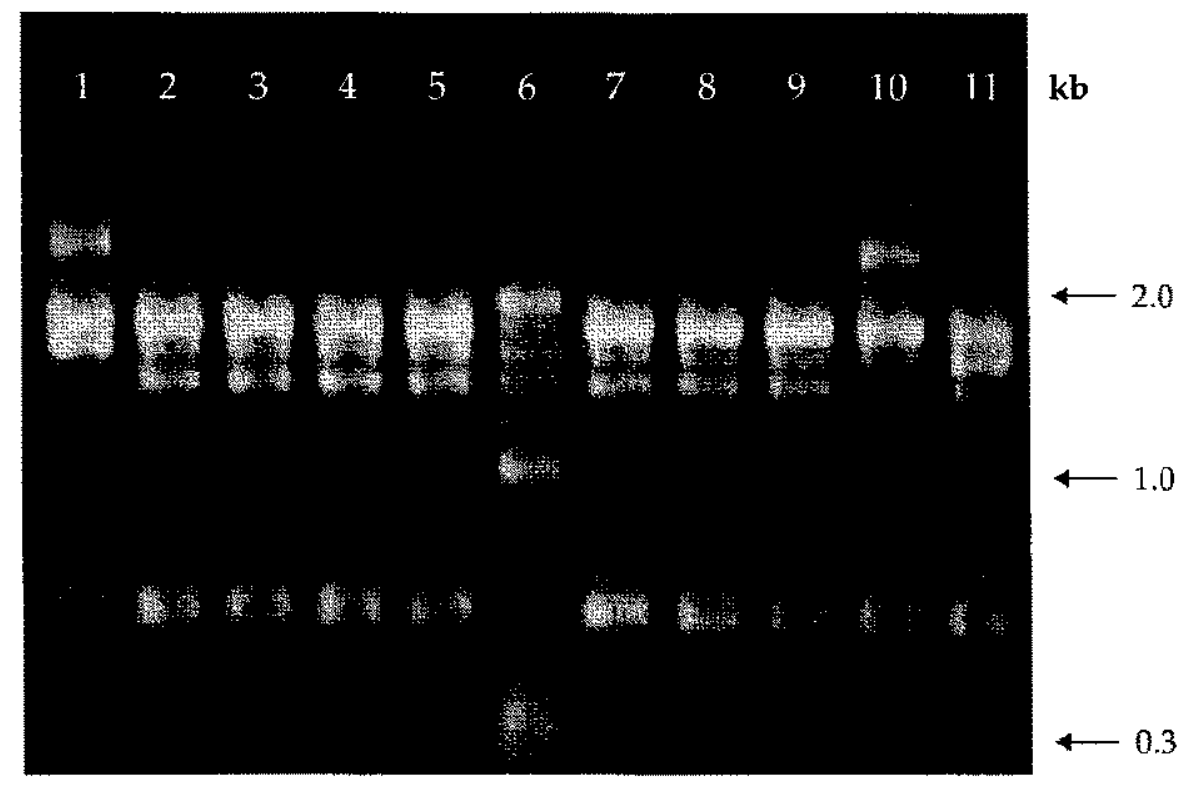

Figura 1. Productos del ADN Polimórfico Amplificado al Azar en cepas de $L$. monocytogenes obtenidos con el cebador M132. Línea (cepa): 1, ma1; 2, m2; 3, ma3; 4, ma4; 5 , ma6; 7, ATCC; 8 , ATCC 7; 9, mcl; 10, mc2; 11, mc3; Línea 6 , Hypperladder II.

tes, pero nuy similares para los 16 aislados, así en la figura 1 se observa 2 perfiles de bandas obtenidos con el cebador M132 para cepas procedentes de leche cruda de Lurín y uno para las de Puente Piedra, estos perfiles son similares a los obtenidos de las cepas de origen clínico.

Los perfiles del RAPD con los 10 cebadores de secuencia arbitraria mostraron un total de 51 fragmentos de ADN entre $0.2 \mathrm{~kb}$ y $3 \mathrm{~kb}$ generando los genotipos denominados I, II y III en este trabajo correspondiendo cuatro, diez y dos cepas respectivamente. Las cepas obtenidas de Puente Piedra perterecen seis y dos a los genotipos II y III respectivamente; las de Lurín cuatro pertenecen al genotipo I y cuatro al genotipo II (Tabla 3). La reproducibilidad de la técnica del RAPD se determinó realizando las reacciones por triplicado y separando los productos amplificados por electroforesis en gel de agarosa.

\section{DISCUSIÓN}

El incremento en los últimos años de brotes de listeriosis y su asociación con el consumo de ali. mentos listos para consumir de diferentes orígenes, han incentivado estudios continuos para una mejor vigilancia y control. Los productos lácteos forman parte de estos alimentos, siendo la leche cruda la de mayor consumo en zonas rurales y urbanas; y la utilizada como insumo para la elaboración de diferentes productos.
Los medios de cultivo Palcam y Oxford son considerados efectivos y selectivos para el aislamiento de L. monocytogenes en alimentos con flora microbiana nativa o contaminante. Sin embargo, el medio Oxford permitió el aislamiento de L. monocytogenes y Listeria spp en muestras de leche cruda, similares resultados obtuvo Harvey y Gilmor (9) al estudia: la presencia de Listeria en leche cruda y productos lácteos en Irlanda.

L. monocytogenes se aisló en el $8 \%$ de muestras analizadas, porcentaje similar a los obtenidos en diferentes investigaciones previamente descritas como Hayes y col., (8) y Lovett y col., (22) en Estados Unidos, donde aislaron esta bacteria en el $12,0 \%$ y $4,2 \%$ de muestras de leche cruda respectivamente. Otros estudios realizados en diferentes paises presentan variación en la incidencia de Listerin en este tipo de alimento, así, en Canadá, Sudafrica y Escocia se describieron $12,4 \% ; 7,6 \%$ y $4,0 \%$ respectivamente (9).

La contaminación de la leche cruda con $L$. monocytogenes proviene del animal y el ambiente; y de otras fuentes de contaminación como son: ensilado, heces, agua, utensilios de ordeño y manipulación lo que permite recomendar hábitos de ordeño y condiciones de higiene controladas a los establos que expenden leche cruda (4).

El uso del RAPD con 10) cebadores de secuencia arbitraria generaron perfiles homogéneos en las 16 cepas de $L$, monocytogenes aisladas de leche 
cruda de las ganaderías de Puente Piedra y Lurin encontrando tan solo tres genotipos. E1 25.0\% (4/ $16), 62.5 \%(10 / 16)$ y $12.5 \%(2 / 16)$ pertenecen a los genotipos I, II y III respectivamente indicando la presencia de tres genotipos en las muestras de leche cruda de las ganaderias de Lima estudiadas siendo el genotipo II, el predominante y el mas disperso por encontrase en muestras de leche de Lurin y Puente Piedra e incluso en las cepas obtenidas de pacientes con listcriosis (Fig 1.).

La mayoría de cebadores descritos en la tabla 1 han sido utilizados por diferentes investigadores para caracterizar cepas de L. monocytogenes aisladas de ambientes, alimentos, y muestras clínicas. El cebador mas descrito y que presentó el mayor número de perfiles es OMP1 $(11,17)$, en este estudio utilizandc el mismo cebador los 16 aislados fueron agrupados en 3 perfiles, apoyando la baja diversidad genética de las cepas de $L$. monocytogenes aisladas.

Conclusiones: se encontró que las 16 cepas de $L$. monocytogenes aisladas de leche cruda se agrupan en tres genotipos, siendo el genotipo II el predominante y disperso, ya que reúne el $62.5 \%$ (10/16) aislados procedentes de Lurin, Puente Piedra e incluso de muestras clínicas de humanos. Estos resultados estimulan la realización de mayores estudios para determinar si este genotipo esta distribuido en productos relacionados o de otros orígenes y si esta asociado a los casos de listeriosis reportados en los hospitales.

\section{AGRADECIMIENTOS}

A la Fundación Internacional para La Ciencia (IFS) por subvencionar parte de este estudio. A quienes nos brindaron las muestras de leche cruda y nos apoyaron en el aislamiento de listeria. A las instituciones que nos proporcionaron las cepas de colección y a J. Pastrana por donarnos las cepas de origen clínico.

\section{REFERENCIAS BIBLIOGRÁFICAS}

1. Farber J.M. y Peterkin P.L. 1991. Listeria monocylogenes a food-borne pathogen. Microbiological Reviews 55, 476-511.

2. Doyle M.P. 1988. Effect of environmental and processing conditions on Listeria monocytogenes. En: Listeria monocytogenes: A Foodborne Pathogen. Food Technology 42, 169-171.
3. Rocourt J. Bille ]. 1997. Foodborne listeriosis. Wld. Hith. Statist. Quart 50, 67-72

4. Nightingale K.K., Schukken Y.H., Nightingale C.R., Fortes E.D., Ho A.J., Her Z., Grohm Y.T., McDonough P.L. Wiedmann M. 2004. Ecology and transmission of Listeria monocytogenes infecting rumiants and in the farm environment. Applied and Environmental Microbiology 70, 4458-4467.

5. Kaplan M.M., Abdusalam M., Bulenga G. 1966. Higiene de la leche (Higiene de la producción, la elaboración y la distribución de la leche). Organización Mundial de la Salud. Enfermedades Transmitidas por la leche. Serie de Monografías. $N^{\circ} 048$.

6. Barza M. 1985. Listeriosis and milk. The New England Journal of Medicine, 312, 438-440.

7. Fleming D.W., Cochi S.L., MacDonald K.L., Brondun J., Hayes P. S., Plikaytis B.D. y col. 1985. Pasteurized milk as vehicle of infection in an outbreak of Listeriosis. The New England Journal of Medicine 312, 404-407.

8. Hayes P.S., Feeley J.C., Graves L.M., Ajello G.W., Fleming DW. 1986. Isolation of listeria monocytogenes from raw milk. Applied and Environmental Microbiology 51, $438-440$.

9. Harvey J. y Gilmour A. 1992. Ocurrence of Listeria species in raw milk and dairy products produced in Northern Ireland. Journal of Applied Bacteriology 72, 119-125.

10.Rea M.C., Cogan T.M., Tobin S, 1992. Incidence of pathogenic bacteria in raw milk in Ireland. Journal of Applied Bacteriology 73, 331-336.

11.Lawrence, L.M., Harvey J., Gilmour A. 1993. Development of a random amplification of polymorphic DNA typing method for Listeria monocytogenes. Applied and Environmental Microbiology 59, 3117-3119.

12.Wiedmann A.J., Ahmad M., Tichy H.V., Schon G. 1994. Characterization of Acinetobacter type strains and isolates obtained from wastewater treatment plants by PCR fingerprinting. Applied and Environmental Microbiology 60, $4066-4071$.

13. Berg D.E., Akopyantz N.S., Kersulyte D. 1994. Fingerprinting microbial genomes using the RAPD or AP-PCR method. Methods of Molecular Cell Biology 5, 174-185 
14.Luoi M., Jayaratne P., Luchinger L., Devenish J., Yao J., Schlech W., Simor A. 1996. Comparison of ribotyping, arbitrarily primed $\mathrm{PCR}$, and Pulse-Field Gel Electrophoresis for molecular typing of Listeria monocytogenes. Journal of Clinical Microbiology. 34, 15-19

15. Gravesen A., Jacobsen T, Moller P.L., Hansen F, Larsen A.G., Knochel S. 2000. Genotyping of Listeria monocytogenes: comparison of RAPD, ITS, and PFGE. International Journal of Food Microbiology 57, 43-51

16. Franciosa G., Tartaro S., Wedell-Neergaard C. 2001. Characterization of Listeria monocytogenes strains envolved in invasive and noinvasive listeriosis outbreaks by PCR-based fingerprinting techniques. Applied Environmental Microbiology 67, 1793-1799.

17.Martinez I., Rovik L., Brox V., Lassen J., Seppola M., Gram L., Fonnesbech- Vogel B. 2003. Genetic variability among isolates of Listeria monocytogenes from food products, clinical samples and processing environments, estimated by RAPD typing. International Joumal of Food Microbiology 84, 285-297
18.FDA-CFSAN. 2001. Listeria monocytogenes. In: Bacteriological Analytical Manual. $9^{\text {th }}$ ed. Capitulo 10.

19.Zavaleta A.I, Martinez-Murcia A.J., RodriguezValera F. 1997. Intraspecific genetic diversity of Oenococcus oeni as derived from DNA fingerprinting and sequence analyses. Applied and Environmental Microbiology 63, 1261-1267.

20. Bubert A., Hein I., Rauch M., Lehner A., Yoon B., Goebel W., Wagner M. 1999. Detection and differentiation of Listeria spp by a single reaction based on Multiplex PCR. Applied and Environmental Microbiology 65, 4688-4692.

21.Jones D., y Seeliger H.P.R. 1992. The Genus Listeria. In Balows A.; Trúper H.G., Dworkin M. Harder W., Scheleifer K.H. The Prokaryotes. A Handbook on the Biology of Bacteria: Ecophysiology, Isolation, Identification, Applications. 2da edición, vol 1, SpringerVerlag, Nueva York. pp. 1595-1616.

22.Lovett J., Twedt R.M. 1988. Listeria. In: Bacteria associated with foodborne diseases. Food Technology 42, 188-191 